\title{
A Hybrid Model for VANET Information Dissemination
}

\author{
Jeyalakshmi Jeyabalan \\ Dept. of Information Technology \\ Rajalakshmi Engineering College \\ Chennai, India
}

\author{
Sree SubhaSoundarajan \\ Dept. of Information Technology \\ Rajalakshmi Engineering College \\ Chennai, India
}

\begin{abstract}
Accidents have been a serious issue of all times. VANET is an emerging mobile adhoc network used in order to improve vehicle and road safety, traffic efficiency, and convenienceas well as comfort to both drivers and passengers. But in VANET, in sparsely populated areas, the messages are not propagated to the neighbors properly, due to fading of messages with distance.There are several solutions to this issue. A Vehicle to Vehicle(V2V) based gossip network may prove effective but if a gossip dies out then it is not fruitful. A V2V store-carry-forward mode of information transfer may be used, but again the results are not guaranteed. The proposed system provides an overview about issues with $\mathrm{V} 2 \mathrm{~V}$ communication and existing solutions in sparsely populated areas, and points to usage of hybrid model with minimal V2V or no $\mathrm{V} 2 \mathrm{~V}$ for sparsely populated areas which uses Vehicle to Road side unit(V2R) or Vehicle- to infrastructure(V2I) which uses existing infrastructure laid for mobile communication and needs very less add-on installations hence proves to be a cost effective solution.
\end{abstract}

\section{Keywords}

Vanet, Adhoc Networks, Wireless Networks, Sparsely Populated Vanet, Gossip Networks

\section{INTRODUCTION}

Safe navigation support through wireless car-to-car and car-tocurb communicationshas become an important priority for car manufacturers as well as transportation authorities. Suchvehicularcommunication networks, which are also referred to as Vehicular Adhoc Networks (VANETs).VANETs provide us a perfect way to collect dynamic trafficinformation and sense various physical quantities related to trafficdistribution with very low cost and high accuracy. This isconsidered essential for achieving automatic and dynamicinformation collection. But there are still issues with parameters like inter-vehicular distance, information dissemination patters and routing protocols. The proposed system takes up the issues pertaining to sparsely populated VANET, where the $\mathrm{V} 2 \mathrm{~V}$ proves to be less effective. So alternative techniques are discussed and a hybrid model for information dissemination is proposed in this system which also proves to be a cost effective solution. The rest of the document is divided into Chapter 2 focusing on the concepts and technologies used in VANET, issues with sparsely populated VANET, and possible solutions for the issues. Chapter 3 illustrates the architecture of the proposed system and the implementation and Chapter 4 discussing the results.

\section{VEHICULAR ADHOC NETWORKS}

VANET is a boon that has proved to provide traffic safety and efficiency in numerous applications.The most important application of VANET is road safety. The VANET disseminates information among On Board Units(OBU) located on vehicles and the Road Side Units(RSU) along with the Infrastructure involved in order to organize ordered flow of data and control among vehicles. The following concepts are the backbone for building the VANET.[1][2]

\section{A. Wireless Access Methods in Vehicular Networks}

2.1.1DSRC/WAVE: [1] Dedicated Short-Range Communication (DSRC) is a short to medium range communication technology operating in the $5.9 \mathrm{GHz}$ range DSRC has two modes of operations: (1) Ad hoc mode characterized by distributed multi-hop networking (vehiclevehicle), (2) Infrastructure mode characterized by a centralized mobile single hop network (vehicle-gateway).

2.1.2 Cellular Networks: Cellular systems have been evolving rapidly to support the ever increasing demands of mobile networking. 2G systems such as IS-95 and GSM support data communications at the maximum rate of $9.6 \mathrm{kbps}$. To provide higher rate data communications, GSM-based systems use GPRS ( $<171 \mathrm{kbps})$ and EDGE $(<384 \mathrm{kbps})$, and IS-95-based CDMA systems use 1xRTT (<141kbps). Now 3G systems support much higher data rate.1 UMTS/HSDPA provides maximum rates of $144 \mathrm{kbps}, 384 \mathrm{kbps}$, and $2 \mathrm{Mbps}$ under high mobility, low mobility, and stationary environments respectively.

2.1.3 WiMAX/802.16e: 802.16e or WiMAX (Worldwide Interoperability for Microwave Access) aims at enabling the delivery of last mile wireless broadband access ( $<40 \mathrm{Mbps}$ ) as an alternative to cable and $\mathrm{xDSL}$, thus providing wireless data over long distances. This will fill the gap between $3 \mathrm{G}$ and WLAN standards, providing the data rate (tens of Mbps), mobility $(<60 \mathrm{Km} / \mathrm{h})$, and coverage $(<10 \mathrm{Km})$ required to deliver the Internet access to mobile clients. In its part, WiBro, developed in Korea based on $802.16 \mathrm{e}$ draft version 3 , provides $1 \mathrm{Km}$ range communications at the maximum rate per user of $6 \mathrm{Mbps}$ and $1 \mathrm{Mbps}$ for down and up links.3 It also supports several service levels including guaranteed QoS for delay sensitive applications, and an intermediate QoS level for delay tolerant application that requires a minimum guaranteed data rate.

2.1.4 WLAN: WiFi or WLAN can also support broadband wireless services. $802.11 \mathrm{a} / \mathrm{g}$ provides $54 \mathrm{Mbps}$ and has nominal transmission range of $38 \mathrm{~m}$ (indoor) and $140 \mathrm{~m}$ (outdoor). Despite its short radio range, its ubiquitous deployment makes WLAN an attractive method to support broadband wireless services on which Internet Access is laid.

\section{B. Broadcasting Techniques In Well Connected Networks}

VANETs ${ }^{[2]}$ are a specific class of ad hoc networks. The commonly used adhoc routing protocols and follow broadcast techniques for delivery. The following discussion explains the techniques used.

2.2.1 Flooding: In simple Flooding scheme, each node in the network will forward the packet exactly one time. This process continues until all nodes in the network receive the packet. 
Simple flooding can be used as a simple protocol for broadcasting and multicasting in ad hoc networks with low node densities and/or high mobility.

\subsubsection{Probabilistic Forwarding : The Probability based} schemes rebroadcast the 1st copy of a received message with predefined probability P. In dense networks, many nodes share similar coverage area. Thus having some nodes to rebroadcast by randomness reduces lots of network overheads without any harming on effectiveness. In case of sparse networks, there is much less shared coverage area so nodes are able to receive packets with high probability.

\subsubsection{Counter-based broadcasting: In Counter-based} broadcasting schemes nodes rebroadcast the message if and only if it received the message from less than $\mathrm{c}$

neighbors. The counter is incremented by one as a redundant packet received. After the timer expires, if the counter is less than a predefined threshold then the node rebroadcast the packet , else it will drop the packet.

2.2.4 Location-based scheme: In Location-based scheme , each node uses an accurate method to determine its location such as a Global Positioning System (GPS). In this scheme the node rebroadcast the message if and only if the additional area covered when the node rebroadcast the message is greater than the threshold A.

2.2.5 Distance based schemes: In Distance based methods nodes rebroadcast the message if and only if the distance to each neighbor that already retransmitted the message id $>$ D.

\section{Problems With V2v Communication}

$\mathrm{V} 2 \mathrm{~V}$ communication is prone to so many issues as discussed below in several scenarios. But the proposed system focuses on only issues pertaining to sparsely populated VANETs. ${ }^{[9]}$

- In $\mathrm{V} 2 \mathrm{~V}$ the connectivity between the vehicles may not be there all the time since the vehicles are moving at different velocities due to which there might be quick network topology changes.

- The anonymity problem: The addresses of vehicles on highways are unknown to each other.

- Periodic broadcasts from each vehicle may inform direct neighbors about its address, but the address-position map will inevitably change frequently due to relative movements among vehicles.

- It is the receiver's responsibility to decide the relevance of emergency messages and decide on appropriate actions.

- Location based broadcast and multicast are the proper communication methods for collision avoidance in $\mathrm{V} 2 \mathrm{~V}$ Communication.

- Without any roadside infrastructure, multihop forwarding must be enabled to propagate the messages or signals

Hence, V2V communication is not very useful in case of Sparsely connected or low density vehicular networks. The proposed system uses a hybrid model that minimally uses V2V communication because of the following issues.

\section{Sparsely Populated Vanets}

The sparsely populated VANETS are those with very less density of traffic due to being situated in rural areas or highly secure lanes. These sparsely populated VANETS are characterised by very less $\mathrm{V} 2 \mathrm{~V}$ communication. Therefore many broadcasting techniques don't prove to be fruitful. The techniques that are used to overcome the issue are stated below.

2.4.1 Store - carry - forward approach: A node v selects a set of candidate forwarders that are closer to the requester or are moving towards the requester. Since it may not be efficient to send a packet to all of the candidate forwarders, the node $\mathrm{v}$ selects a subset of candidate forwarders, based on the amount of knowledge it has.

2.4.2 Gossip Networks : SCT Approach Using greedy routing, a message is forwarded to the neighboring node which is "closest" to the destination. The notion of a node's location (and hence "closeness") may be determined by its physical location, as ingeographic routing used by ad hoc networks. Location may also be an entirely artificial construct as in small world routing and distributed hash table.

But there are various shortcoming in these methods of communication. Chapter 5 states the comparative analysis of various techniques as compared to the proposed system. The system architecture of the proposed system to handle effective communication in sparsely populated region is discussed below.

\section{SYSTEM ARCHITECTURE}

The proposed system uses a hybrid model with V2I and V2R widely used in application with less or no $\mathrm{V} 2 \mathrm{~V}$ communication since the scenario is a sparsely populated VANET. The dedicated patrol vehicles are employed to collect information about the scanned road space. The proposed system uses the existing cellular infrastructure in order to carry the V2I communication. This saves the cost involved in laying the infrastructure for traffic management. The system uses short range signals for establishing the ad hoc network between the Road side unit and the vehicle. The system makes use of already existing infrastructure like the Traffic Policing infrastructure that is well established. The information regarding any Toll Payment, Speed Limits or Road Blocks is intimated by the Traffic Patrol vehicle to the RSU. The vehicles are equipped with the subscriber identification module in order to be uniquely identified by the BSC and MSC

- This helps the cellular Infrastructure units totrack any vehicle that is involved in any malicious actions.

- This also helps to monitor the traffic pattern of the vehicle and $\log$ the information.

- There can be better automated traffic model analysis.

- This also helps to avoid any accidents that could have been caused in the vehicle had traveled without warning of the oncoming disasters. 


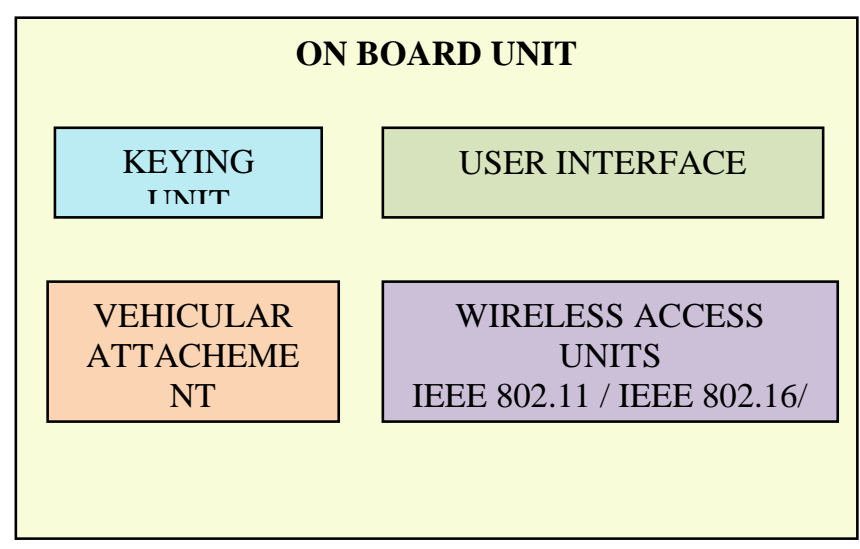

Fig. 1. Design of the On Board Unit

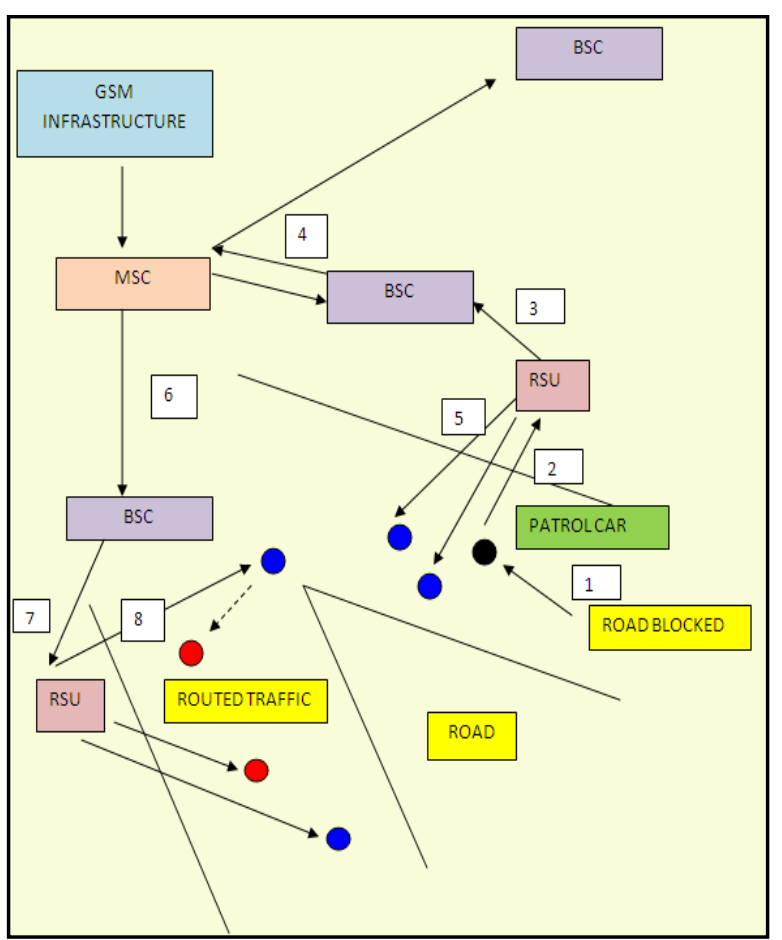

Fig. 2. Proposed System for Traffic Management

In the proposed system the following is the flow of action.

- The patrol vehicle encounters the hot spot that involves issues.

- The disasters are intimated by the patrol vehicles to the RSU.

- The RSU communicates the same to the Base Station and the Mobile Switching center.

- The RSU intimates of issues or any information to all vehicles in the VANET.

- The MSC communicates the information to all the other Base Stations in the locality.

- The other Base Stations communicate the message to all the RSU in the related locations.
- The related RSU now performs any action like routing of traffic in alternate direction in order to overcome the situation

The On Board Units are placed in every vehicle, and their design is explained below. The OBU are equipped with the module to perform wireless communication via the protocols like wi-fi, wimax or wave. The OBU has a display unit to communicate the messages from the RSU. There is support for keying any information to RSU in case of any issue. ( The proposed system assumes that this capability is provided with the patrol cars to overcome false positive alerts from other vehicles and to overcome the security issues). The OBU has the attachment module to the vehicle in order to establish the control.

\section{IMPLEMENTATION}

The sparsely populated VANET involves less communication amid vehicles. This scenario is implemented using VNSim and the results are discussed below. Figure 3 and 4 characterize the densely populated VANETs(DPV) while Figure 5 and 6 characterize the sparsely populated VANETs(SPV).

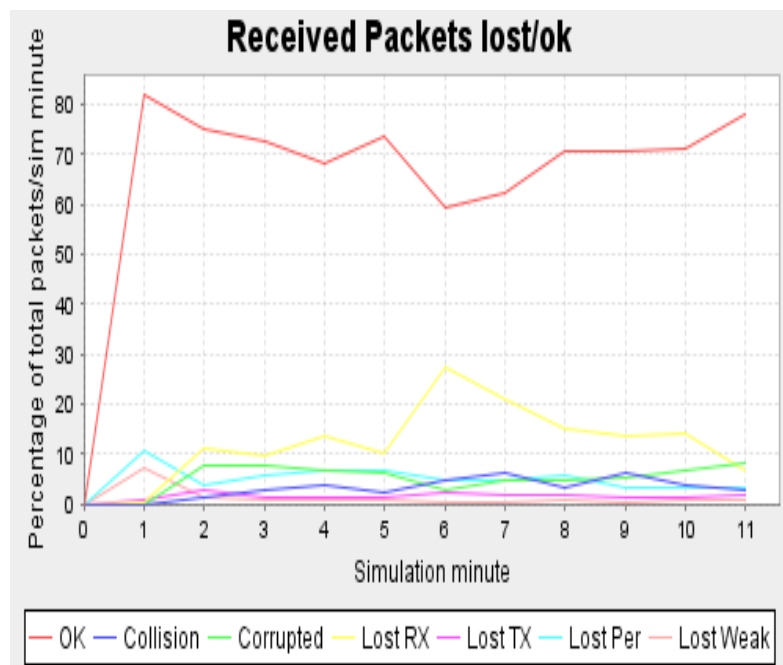

Fig. 3. Packet transmission statistics in DPV

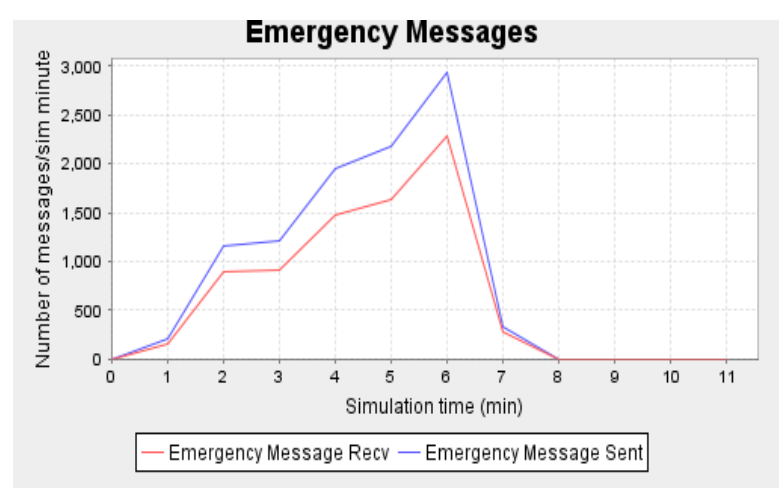

Fig. 4. Emergency Messages Communication Statistics in DPV 


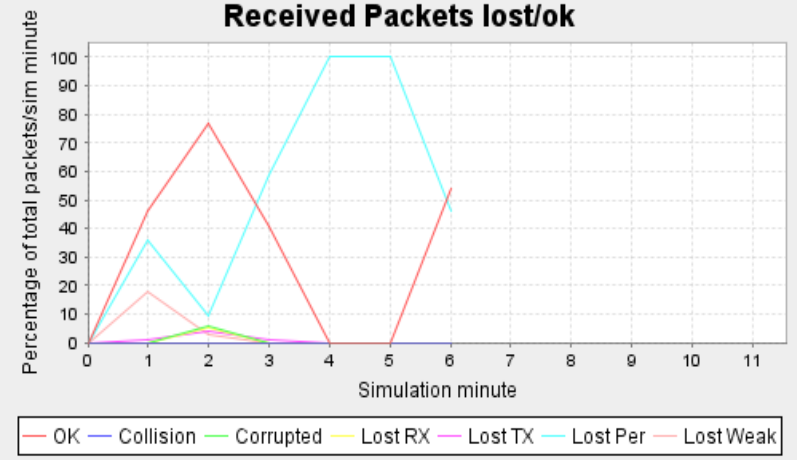

Fig. 5. Packet transmission statistics in SPV

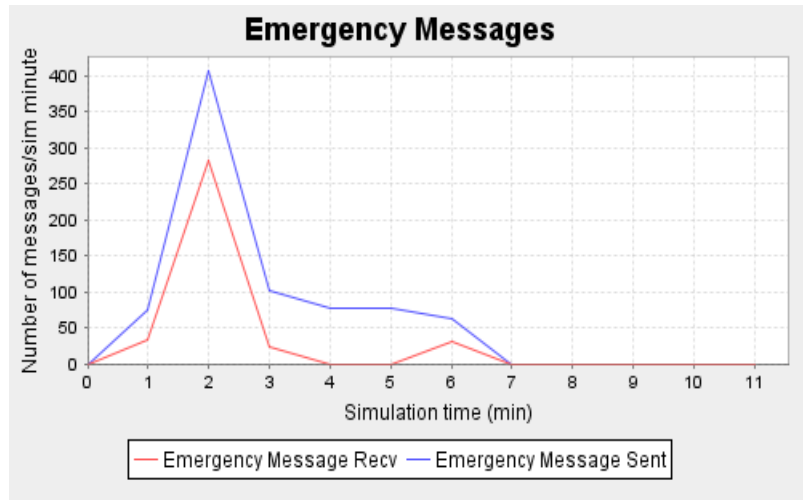

Fig. 6. Emergency Messages Communication Statistics in SPV

The graphs show that, the reliability of reception of both normal and emergency messages using probabilistic forwarding is very less in SPV and high in DPV. This states that there is need for an alternate technique which will have to patch the system to provide effectiveness.

The VANET simulation for the hybrid model on a SPV is performed in the movsim. The simulation screenshots show that the road blocks are leading to a traffic bottleneck. The RSU on proper intimation as per the flow of information suggested in the proposed architecture, routes the traffic in different direction to overcome the bottleneck.

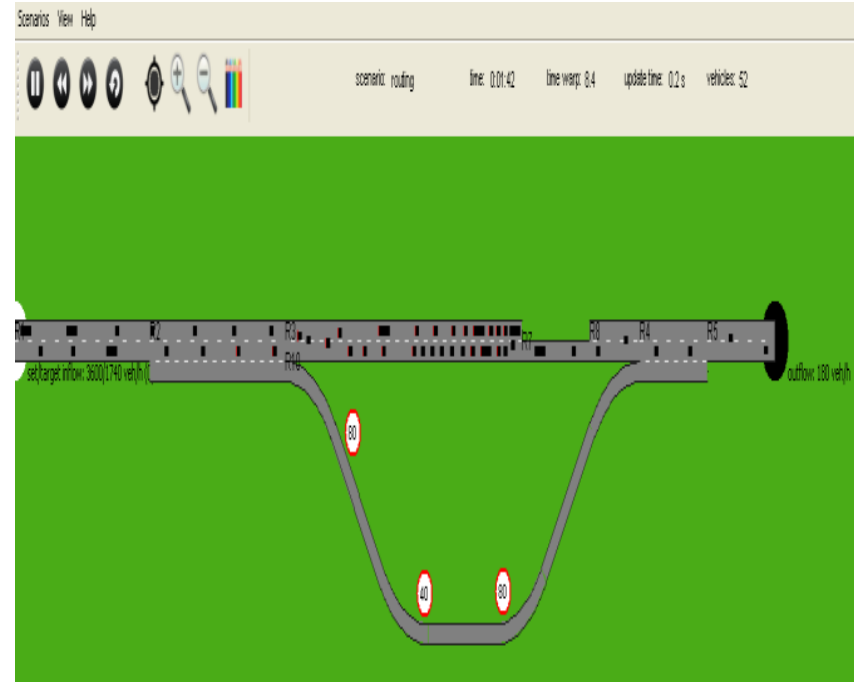

Fig. 7. Road Block leading to a Bottleneck

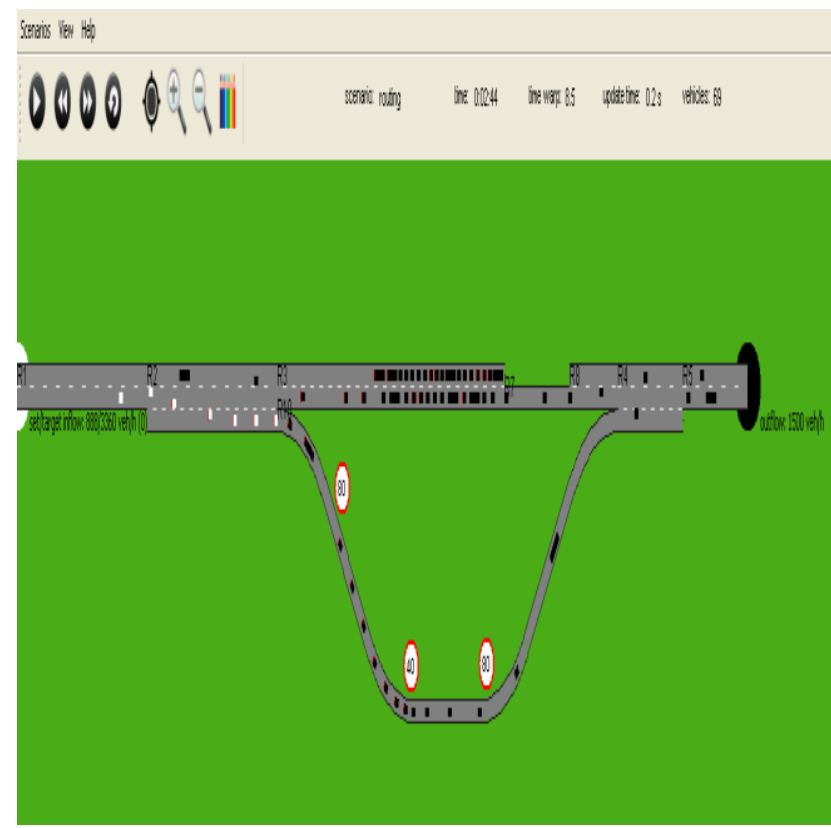

Fig. 8. Routing in order to overcome the Bottleneck.

\section{PERFORMANCE ANALYSIS}

The proposed system is a effective mode of information dissemination compared to the flooding based broadcast methodologies or SCT / Gossip Networks that are fixes for the sparsely populated VANET issues.

The table 1 shows the effectiveness of the hybrid communication as compared to existing solutions to sparsely populated VANET issues. ${ }^{4][5][6][7]}$ 
Table 1: Comparative analysis between existing and proposed system for handling isssues in SPV

\begin{tabular}{|c|c|c|c|}
\hline & Techniques & Strengths & Weakness \\
\hline 1 & $\begin{array}{l}\text { Gossip Networks in } \\
\text { V2V }\end{array}$ & $\begin{array}{l}\text { - Some nodes receive a packet } \\
\text { multiple times. But not as much as } \\
\text { flooding. } \\
\text { - Efficient use of bandwidth } \\
\text { - Broadcast storm problem is } \\
\text { avoided. }\end{array}$ & $\begin{array}{l}\text { - Attempts are needed to accommodate the heavy } \\
\text { traffic resulting from gossiping by probabilistically } \\
\text { forwarding data packets, effectively minimizing the } \\
\text { number of forwarding nodes. } \\
\text { - Additional effort is needed to compensate for packets } \\
\text { that seem to be dying out by rebroadcasting them, } \\
\text { thereby maximizing the dissemination coverage. } \\
\text { Despite the optimizations, in routing protocols that } \\
\text { use flooding, many routing messages are propagated } \\
\text { unnecessarily. }\end{array}$ \\
\hline 2 & $\begin{array}{l}\text { SCT Approach in } \\
\text { V2V }\end{array}$ & $\begin{array}{l}\text { - } \begin{array}{l}\text { Overcomes } \\
\text { connectivity. }\end{array} \\
\text { - } \quad \text { Saves node and network resource. }\end{array}$ & - $\quad$ No delivery effectiveness \\
\hline 3 & $\begin{array}{l}\text { Hop Count Based } \\
\text { Limited Forwarding } \\
\text { Approach in V2V }\end{array}$ & $\begin{array}{l}\text { - An advantage of the hop-count- } \\
\text { limited probabilistic forwarding } \\
\text { scheme over many other } \\
\text { probabilistic forwarding schemes } \\
\text { is that, given a constant } H \text {, it has a } \\
\text { constant per message cost, which } \\
\text { is necessary to achieve ultimate } \\
\text { scalability: given a constant per } \\
\text { node message rate, the per node } \\
\text { forwarding overhead is kept } \\
\text { constant as the network size } \\
\text { increases. }\end{array}$ & $\begin{array}{l}\text { - A forwarding decision based on comparing the direct } \\
\text { forwarding qualities of nodes } i \text { and } j \text { cannot guarantee } \\
\text { a good forwarding. (1) The forwarding quality of } j \\
\text { being better than } i \text { does not necessarily mean that } j \text { is } \\
\text { a good forwarder. (2) Even though the quality of } j \text { is } \\
\text { high, } i \text { might encounter better nodes in the near } \\
\text { future.(3) Similarly, even though the quality of } j \text { is } \\
\text { lower than } i, j \text { might still be the best forwarder that } i \\
\text { could encounter in the future. } \\
\text { Effective only if indirect delivery probability also is } \\
\text { taken into account. } \\
\text { The quality of a node is a constant regardless of two } \\
\text { important states of the copy: remaining hop-count and } \\
\text { residual lifetime. }\end{array}$ \\
\hline 4 & $\begin{array}{l}\text { Hybrid } \quad \text { Model } \\
\text { following } \quad \text { Wi-Fi } \\
\text { Routing Techniques }\end{array}$ & $\begin{array}{l}\text { - There is effective delivery. } \\
\text { - No complexity involved in } \\
\text { calculating hops or distance or } \\
\text { location based relativity with } \\
\text { vehicles. }\end{array}$ & $\begin{array}{l}\text { - The cost of laying the initial infrastructure may be } \\
\text { high compared to V2V based techniques, but the } \\
\text { advantages are comparatively higher. }\end{array}$ \\
\hline
\end{tabular}

The table 2 below shows the effectiveness of the hybrid communication as compared to various broadcast techniques that use flooding methodology. ${ }^{[4][5][6][7]}$

Table 2. Comparative Analysis between various information dissemination techniques

\begin{tabular}{|c|c|c|c|c|c|}
\hline $\begin{array}{l}\text { Information } \\
\text { Dissemination } \\
\text { Technique }\end{array}$ & Flooding & $\begin{array}{l}\text { Probability } \\
\text { based } \\
\text { methods }\end{array}$ & $\begin{array}{l}\text { Location } \\
\text { based } \\
\text { methods }\end{array}$ & $\begin{array}{l}\text { Distance } \\
\text { Based \& } \\
\text { Location } \\
\text { based }\end{array}$ & $\begin{array}{l}\text { Hybrid } \\
\text { Model }\end{array}$ \\
\hline $\begin{array}{l}\text { Broadcast } \\
\text { storms }\end{array}$ & Severe & Limited & Limited & Controlled & $\begin{array}{l}\text { Highly } \\
\text { Controlled }\end{array}$ \\
\hline Mobility & $\begin{array}{l}\text { Highly } \\
\text { Robust }\end{array}$ & Robust & Robust & Robust & Robust \\
\hline Rebroadcasts & All & Most & Constant & Constant & Constant \\
\hline $\begin{array}{l}\text { Retransmission } \\
\text { Recovery }\end{array}$ & No & No & Limited & Limited & No \\
\hline Reliability & Very High & High & High & High & Very High \\
\hline Redundancy & Very High & Restricted & Low & Low & Very Low \\
\hline Scalability & Restricted & $\begin{array}{l}\text { Partially } \\
\text { Restricted }\end{array}$ & High & $\begin{array}{l}\text { Partially } \\
\text { Restricted }\end{array}$ & High \\
\hline
\end{tabular}

\section{CONCLUSION}

The proposed system aims at using the existing infrastructure along with few enhancements in order to save cost and avoid accidents. The proposed system uses a hybrid model that minimally uses $\mathrm{V} 2 \mathrm{~V}$ communication because of the issues that prevail with $\mathrm{V} 2 \mathrm{~V}$ in sparsely populated network scenario as briefed in Chapter 2. The proposed system proposes usage of both long range and short range communication in order to offer contingency based information communication. This proves to be more effective and reliable mode of communication compared to the existing systems.

\section{REFERENCES}

[1] SheraliZeadally, Ray Hunt, Yuh-Shyan Chen, Angela Irwin - Aamir Hassan, Dr. T. Karthikeyan and J. Geetha, "Vehicular ad hoc networks (VANETS): status, results,and challenges," TelecommunSyst, DOI 10.1007/s11235-0109400-5.

[2] Uichin Lee, Ryan Cheung, Mario Gerla, "Emerging Vehicular Applications", 2011

[3] Avanti Chimote ,CharanHebri, Kuppuraj ,GunasekaranSandeep N L,SaravananSathanandaManidas,"Collision Avoidance in Vehicular Networks

[4] Cong Liu and Jie Wu, "An Optimal Probabilistic Forwarding Protocol in Delay Tolerant Networks", MobiHoc'09, May 18-21, 2009 
[5] Sara Najafzadeh, Norafida Ithnin, Shukor Abd Razak," A new routing protocol for Vehicular Ad Hoc Networks",2011

[6] Zygmunt Haas ,Joseph Y. Halpern ,Li Li, “Gossip-Based Ad Hoc Routing", 2012

[7] Albana Gaba_, Spyros Voulgaris_, Konrad Iwanickiy and Maarten van Steen," Revisiting Gossip-based Ad-Hoc Routing”, 2010
[8] Koosha Paridel, Yolande Berbers, Josip Balen, Goran Martinovic, "VVID: A Delay Tolerant Data Dissemination Architecture for VANETs Using V2V and V2I Communication, MOBILITY 2012 : The Second International Conference on Mobile Services, Resources, and Users

[9] Avanti Chimote ,CharanHebri, Kuppuraj ,GunasekaranSandeep N L,SaravananSathanandaManidas , "Collision Avoidance in Vehicular Networks. 